\title{
Analysis and Design of Orchestral Instruments Sales Management System Based on B/S Structure
}

\author{
Ying Yue \\ Basic Teaching \& Research Department, Bohai University, Jinzhou, 121013, China \\ yongyuanaizhemama@163.com
}

Keywords: B/S structure; .NET platform; orchestral instrument; management system; feasibility analysis;

\begin{abstract}
With the continuous development of world music and music educational undertakings, orchestra has gradually appeared in various forms of musical performances; the kind of orchestral instruments also became more and more, so the design and implementation of an orchestral instruments sales management system is important. Firstly, based on the study of the B/S structure software system development framework under the .NET platform, mainly describes the B/S three-tier structure and its advantages; secondly, studies the feasibility analysis of orchestral instruments management system, mainly includes two aspects that are economical feasibility and technical feasibility; finally studies the summary design of the system, namely the overall design, detailed describes the functional flow diagram of the system. Orchestra instruments make the form of music become more diversely, break the tradition of regular performances, the analysis and design of orchestral instruments sales management system promotes the rapid development of music.
\end{abstract}

\section{Introduction}

Orchestral instruments include five classes that are string, copper tube, woodwinds, plucked string and percussion. In which stringed instruments is vibrating strings and pronunciation, there is bowed stringed instrument, plucked stringed instrument and hit stringed instruments three kinds, commonly stringed instrument is bowed stringed instrument, bowed stringed instruments have violin, viola, cello and double bass four kinds; brass including trumpet , French horn, trombone and tuba; woodwind group includes flute, clarinet, oboe, bassoon, etc.; plucked string mainly include harp; percussion includes timpani, triangle, xylophone, glockenspiel, castanets, sand hammer, snare drum, cymbals, gongs and the like. The traditional Chinese orchestral instruments include gehu, zhonghu, gaohu, erhu, violin, harp, lute, etc., they has made tremendous contributions to our musical development [1].

.NET platform provides a good foundational platform for the development of the system, .NET framework is an executed environment which has multi-language component development, it provides a unified programming environment across languages, mainly consists of three parts which are the common language runtime (CLR), a unified hierarchical library (FCL) and ASP.NET programming framework. ASP.NET is a programming framework established in the common language runtime, provides a Web application model, which consists of a set of controls and a basic structure, it can generate a powerful Web applications on server [2]. .NET framework implements language development, code compilation, component configuration, program running, object interactions, and other functions, provides a managed, secure and efficient execution environment for Web services and general applications [3]. .NET is structured, but also is hierarchical; each layer of .NET framework is a abstract layer. .NET is modular, it can be divided into several modules, and each module has its own separate functions.

\section{B/S Structural Framework}

$\mathrm{B} / \mathrm{S}$ structure namely is the browser and server structure. It rises with the of Internet technology, it is a change or improvement for $\mathrm{C} / \mathrm{S}$ structure, meanwhile, has its own unique advantages with all 
the advantages of $\mathrm{C} / \mathrm{S}$ structure. In this structure, the user working interface is achieved through the WWW browser, small part of business logic are realized in browser, but the main business logic are implemented on the server side [4]. The main features of B/S structure is the strong distribution, easy to maintain, simple develop and share ability, the overall low cost. Advantages of this structure are low cost, low maintenance; achieve cross-platform operation; reduce database concurrent users.

B/S 3-tier architecture mainly includes the presentation layer (Presentation), business layer (Business Logic), the data layer (Data Service). Presentation layer consists of UI (User Interface) and the UI control logic. UI is a client browser, responsible for making service requests from a Web browser to a Web server on the network. UI control logic handles data exchange between UI and business layer, the control flow between the UI states, is also responsible for simple data validation and formatting and other functions; business layer encapsulates the actual business logic, including data validation, transaction processing, permissions processing and other business-related operations, is the core of the application system; data layer is composed of business data access layer and data access layer, business data access layer provides a minimum mode of operation for interacting with data sources, the role of the data access layer provides an interface for external access for the data source [5].

For application system based on B/S structure, the main function of the system and application logic are processed in the application service layer, in an object-oriented system, the application service layer must process the data, the organized manner and implemented manner of application logic. The nature of the application service layer is the application framework, established the application logic layer for logic processing on all of its packaging systems. In the application system framework, application service layer is divided the data presentation layer, data access layer, the application rules layer and application appearance layer[6], the relationship among application service layer at all levels is shown in Fig. 1.

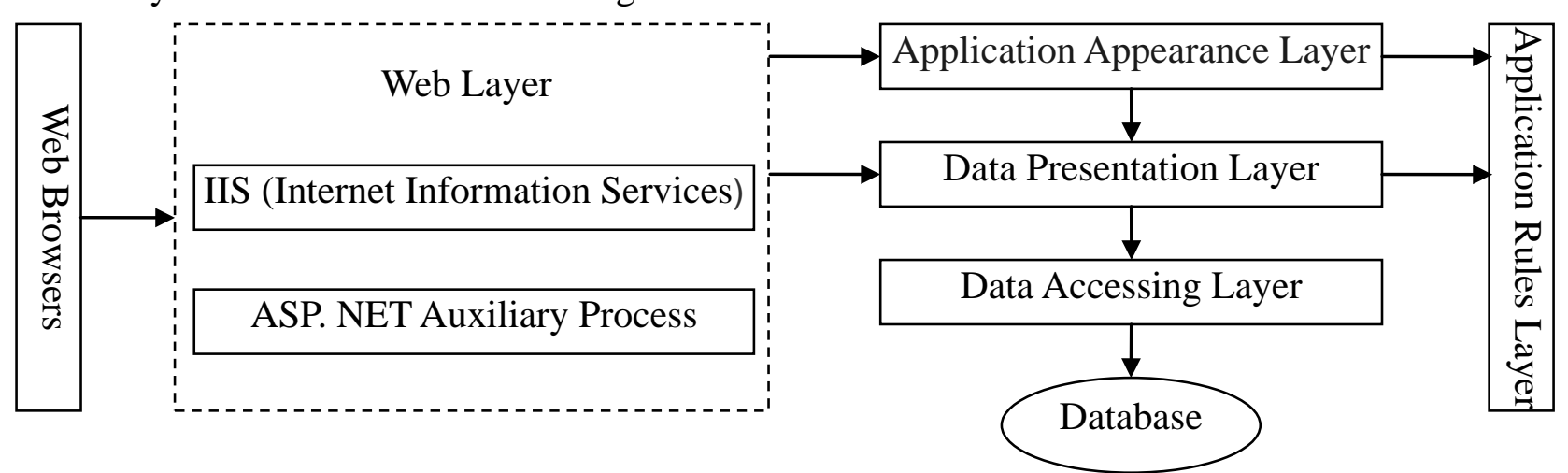

Fig. 1. B/S system development framework diagram under .NET platform

\section{Feasibility Analysis}

Feasibility analysis is analysing and comparing the system's main content and related conditions from the aspects of technical, economic and others, to determine the condition in the development of the system. The feasibility study mainly includes technical feasibility, economic feasibility, tissue viability, social feasibility, control of risk factors feasibility and so on. Feasibility analysis should have predictability, fairness, reliability, and scientific characteristics [7]. Feasibility Analysis of orchestral instruments management system designed in this paper mainly describes the technical feasibility and economic feasibility.

Economic feasibility analysis divided into cost analysis and benefit analysis, by comparing demonstrating system development is right or not on the economic cost. The development of orchestral instruments sales management system based on B/S structure mainly obtain better economic and business management benefits. Development costs include the purchase of hardware and software systems and related equipment costs, installation costs of system development, operation and maintenance costs as well as personnel training costs. Equipment needed system developed in this paper is mainly hosts, servers and networking equipment, using virtual hosts, 
equipment costs will be greatly reduced; system client is browser, using B/S mode development and maintenance costs are also lower [8]. The functions realized orchestral instruments sales system provides a comprehensive information platform for orchestral instruments sales, significantly improved the economics of orchestral instruments dealer.

Technical feasibility refers to achieving the feasibility of the system development based on existing technology. In software development, orchestral instruments management system designed in this paper uses more popular B/S three-tier structure model, in close connection with the Internet/Intranet technology; system development using ASP.NET programming technology, technology is mature and advanced. Database uses SQL Server technology that can handle large amounts of data; In terms of hardware, the storage capacity of the computer, computing speed and functionality is increasing, the ability and quality of network communications equipment also continued to strengthen. Therefore, under the current conditions, hardware and software technology completely meet the needs of system development [9].

\section{Summary Design}

Summary design is also known as the overall design, namely the design of the global problem, which is the overall treatment program of the system design. In software engineering summary designs includes computer configuration design, system module design, database and document design, code design, system reliability and internal control design and so on. The overall design of this study is mainly the functional design of orchestral instruments sales management system. based on B/S structure of orchestral instruments complete sales management system designed in this article mainly achieved orchestral instruments sales management, includes instruments information management, musical instruments purchase management, order information management, instrument inventory management, financial information management, maintenance management instruments, system management and user management. System functional flowchart is shown in Fig. 2.

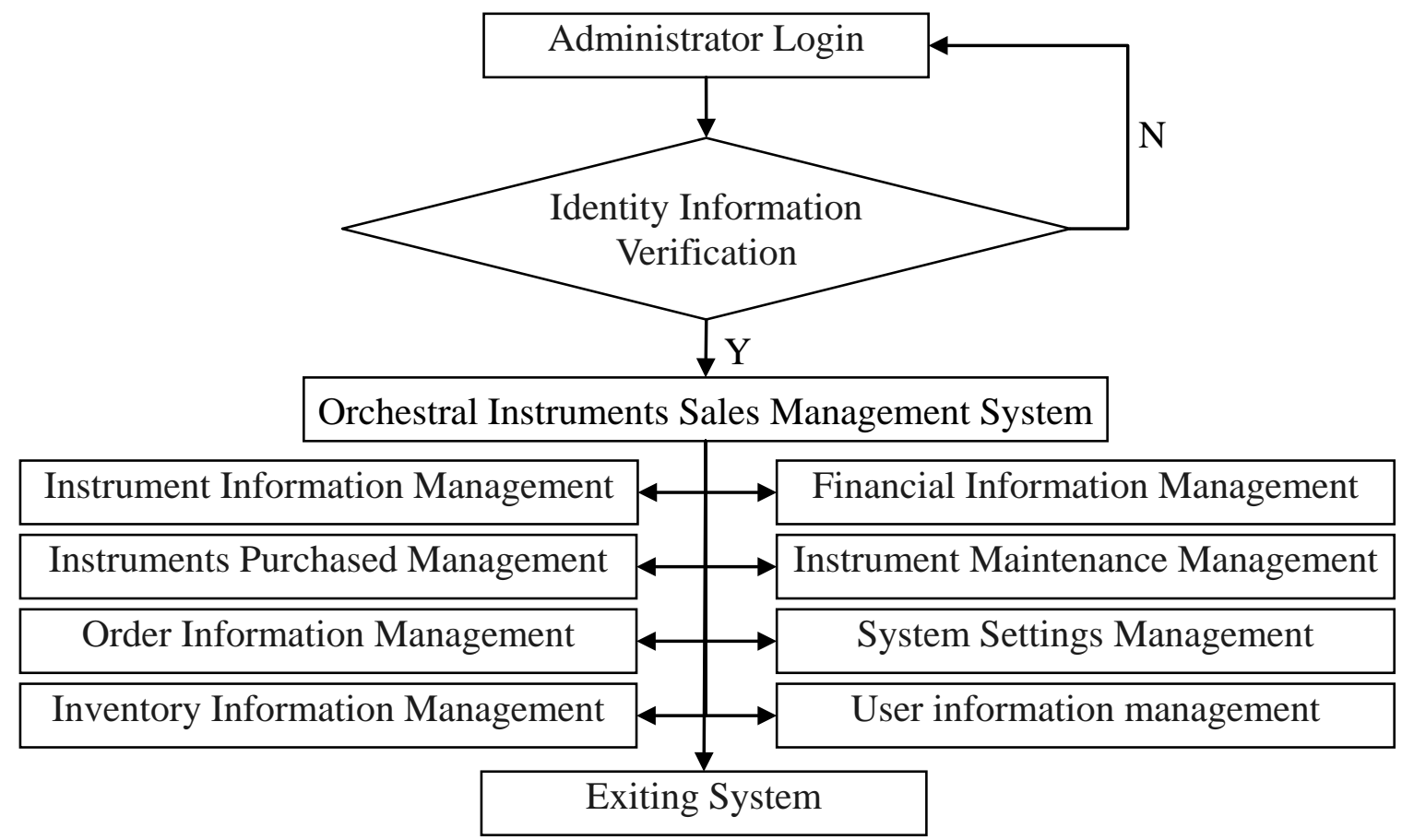

Fig. 2. System functional flowchart

Instruments information management module mainly manages the information of orchestral, which includes instrument name, instrument numbers, instrument usage, instrument classification, instrument origin, instrument price, etc.; instruments purchase management includes the vendor information, instrument purchase price, the number of instruments purchased and so on; Order information management module according to historical inquiry and statistics to develop marketing 
strategies; inventory management mainly manage instruments inventory, includes inventory number of musical instruments, musical instruments types, etc.; financial information management includes accounts receivable management and accounts payable management, accounts receivable generally generated with instruments sales, accounts payable generally produced with the purchase of musical instruments; instruments maintenance management mainly maintenance and repair instruments; system management mainly includes system maintenance, settings, and some customer feedback management; users management is to manage the user information who login system, includes permission settings and so on.

\section{Conclusion}

Orchestral instruments is an important branch within the instruments family, in a modern light music and even classical music, almost all of the lyrical melody are played by the orchestra, the dominant position of orchestral instruments in the music is growing stability [10]. The rapid development of computer technology further spread the fashion trends of orchestral, the paper analyzed the design orchestral sales management system using B/S three-tier structural framework, effectively implements the system relatively independent of each functional layer, makes the system have more great flexibility, scalability and maintainability; and system hierarchical management makes hierarchical distributed deployment become reality, it can greatly improve the performance and safety of the system to meet the growing demand for B/S mode application system [11]. Therefore orchestral instruments sales management system not only meets people's purchasing needs for orchestra, but also produces great economic benefits for the instruments dealers, has a great significance to the development of world music.

\section{References}

[1] Baidu Baike, "namespace," http://baike.baidu.com/view/2737437.htm?fr=aladdin, 2014-10-10.

[2] Q. H. Tong, Q. Mei, "Framework of B/S Mode System Based on .NET Platform," Microcomputer Development, vol. 14, no. 8, pp. 62-63, 2004.

[3] C. M. Wu, "Research of Multi-layer Architecture Based on .NET Platform," Master's Degree of University of Wuhan Science and Technology, 2012.

[4] Z. R. Zhang, "Discussion on C/S Structure and B/S Structure and Future Development," Science \& Technology Information, vol. 25, no. 17, pp. 94, 2008.

[5] W. H. Zhao. Q. C. Ming, "Research on Software Design Based on B/S 3-Tier Structure," Research and Exploration in Laboratory, vol. 30, no. 7, pp. 65-66, 2011.

[6] J. D. Chen, G. W. Cao, "The Design and Achievement of Enterprise Stock-sell-storage Management System Based on Browser/Server Pattern," Modular Machine Tool \& Automatic Manufacturing Technique, vol. 48, no. 5, pp. 110-112, 2006.

[7] Baidu Baike, "namespace," http://baike.baidu.com/view/228933.htm?fr=aladdin, 2014-10-10.

[8] M. Y. He, "Design and Realization of Medicine Sales Management System Based on B/S," Master's Degree of Jilin University, 2006.

[9] F. Niu, "Design and Implementation of Automobile Sales Management System Based on ASP.NET," Master's Degree of University of Electronic Science and Technology of China, 2013.

[10] Q. Y. Chen, "Research on Position of Orchestral in the Music Development of Our Country," Jiannan Literature, vol. 35, no. 10, pp. 314, 2011.

[11] L. X. Xu, "Design and Implementation of B/S Three-tier Structure Based on ASP.NET Technology," Computer Knowledge and Technology, vol. 15, no. 1, pp. 34-35, 2008. 